\title{
Delirium en adultos mayores y anestesia general guiada por electroencefalografía. Estudio clínico piloto, randomizado, prospectivo
}

\section{Clinical pilot study in elderly patients under general anesthesia guided by EEG}

\author{
Jimena Rodríguez ${ }^{1, *}$, M. Serra ${ }^{1}$, Cristina Rondón ${ }^{1}$, M. José Rodríguez¹, Sebastián Muñoz², Mario Neira ${ }^{1}$, Katherine Dziejarski², \\ Pablo de Carolis Arena², Javier González de la Vega ${ }^{3}$, Hugo Loyola ${ }^{a}$ \\ 1 Equipo de Anestesiólogos Clínica INDISA Universidad Andrés Bello, Santiago, Chile.
}

Conflictos de interés: Ninguno de los autores tiene conflictos de interés, ni actividad comercial de algún tipo asociada con los temas de este trabajo de investigación clínica.

No se recibió ningún tipo de financiamiento ni ayuda financiera para la realización de este estudio. Se contó con la colaboración del desinteresado trabajo de todos los colegas anestesiólogos de Indisa.

Fecha de recepción: 12 de abril de 2021 / Fecha de aceptación: 25 de junio de 2021

\begin{abstract}
Introduction: Perioperative delirium (PD) is a serious cerebral complications in the elderly. Over the past decade, some evidence has emerged that monitoring depth of general anesthesia (GA) with electroencephalography (EEG) could help decrease these complications. Methods: 200 patients over 65 years of age undergoing noncardiac surgery under general anesthesia were monitored with Sed line (SL) and spectrogram. Patients were randomized to receive GA with and without Sed Line and Spectrogram. In group 1 the SL was adjusted between 25 and 50 and SEF between 8 and 14. In group 2 without SL, anesthesia was adjusted according to clinical and traditional hemodynamic parameters. The appearance of PD was evaluated with the postoperative CAM test. Results: 7 patients presented PD. 6 of these patients belong to the group where no EEG control was added and 1 patient presented PD in the EEG group. A reduction of DP from $5.9 \%$ to $1.0 \%$ was observed when using EEG. There is no significant difference between groups ( $p$-value $=0.1305$ ). Conclusions: In the study population, EEG-guided general anesthesia shows a trend to decrease PD, in relation to general anesthesia without EEG.
\end{abstract}

Key words: Perioperative delirium, EEG.

\section{RESUMEN}

Introducción: El delirium perioperatorio (DP) es una grave complicación cerebral en el adulto mayor. Durante la última década han surgido algunas evidencias de que la monitorización de la profundidad de la anestesia general (AG) con electroencefalografía (EEG) podría ayudar a disminuir esta complicación. Métodos: Se monitorizó con Sed line (SL) y espectrograma a 200 pacientes mayores de 65 años sometidos a cirugía no cardiaca bajo anestesia general. Se randomizaron los pacientes a recibir AG con y sin Sed Line y Espectrograma. Se ajustó en el grupo 1 el SL entre 25 y 50 y SEF entre 8 y 14 . En el grupo 2 sin SL, la anestesia se ajustó de acuerdo a clínica y parámetros hemodinámicos tradicionales. Se evaluó la aparición de DP con test CAM posoperatorio. Resultados: Siete pacientes presentaron DP. Seis de estos pacientes pertenecen al grupo donde no se agregó control con EEG y 1 paciente presenta DP en el grupo con EEG. Se observó una reducción de DP desde 5,9\% a 1,0\% cuando se utiliza EEG. No hay diferencia significativa entre grupos ( $p$-value $=0,1305)$. Conclusiones: En la población estudiada, la anestesia general guiada por EEG, muestra una tendencia a la disminución del DP, en relación con anestesia general sin EEG.

Palabras clave: Delirium perioperatorio, electroencefalograma. 


\section{Introducción}

as alteraciones cognitivas posoperatorias se conocen desde hace más de 60 años y se ha tratado de asociar la anestesia general con el daño cerebral posoperatorio. Estas alteraciones cognitivas son principalmente la disfunción cerebral posoperatoria (DCPO) y el delirium posoperatorio (DP)[1],[2].

El DP es una forma aguda y transitoria, de disfunción cerebral cuyos síntomas son desorientación, alteración de la atención, memoria y ciclo circadiano. Se produce en el adulto mayor y es la más frecuente complicación cerebral que aparece en el adulto mayor[3]. Estudios recientes sugieren que la DCPO y el DP son más frecuentes después de anestesia general[1].

Existe evidencia en animales que sugiere que dosis habituales de anestésicos generales producen alteraciones de memoria y aprendizaje. Esto se asocia con hiper-fosforilación Tau, activación de caspasa 3 y producción de proteínas $B$ amiloide en el cerebro, los tres procesos asociados a enfermedad de Alzheimer y a toxicidad cerebral. Estos datos aún no son concluyentes en humanos pero hay evidencia de que los anestésicos pueden contribuir a daño cerebral posoperatorio[4],[5],[6],[7],[8].

La anestesia inadecuada ya sea por profundidad excesiva o insuficiente se ha asociado con outcomes negativos, como la DCPO, el DP y awareness que es el término aceptado para la complicación anestésica por dosificación insuficiente[8],[9].

Los pacientes que presentan DP tienen mayor mortalidad y una mayor incidencia de complicaciones posoperatorias como Infarto miocárdico, neumonía, insuficiencia renal, infecciones, prolongación del tiempo de hospitalización y más frecuente traslado de estos pacientes a unidades de cuidados cróni$\cos [3],[10]$.

El cerebro es el órgano blanco de la anestesia general por lo que se debería monitorizar especialmente en este tipo de anestesia. Desde 1937 se ha intentado incorporar la monitorización guiada por electroencefalografía (EEG) a esta técnica de anestesia. Actualmente, contamos con EEG digital que ha permitido el desarrollo de monitores de profundidad anestésica en la práctica de la anestesia general, que podrían mejorar el manejo anestésico y probablemente el outcome del paciente[5],[8],[9],[10].

La EEG se empezó a utilizar como monitor en 1970, cuando Bickford describió el uso de la transformación rápida de Fourier (FFT) como una herramienta de análisis del EEG.

FFT permite un análisis cuantitativo y estadístico de la señal de EEG como una forma numérica de poder y frecuencia[11],[12]. Este desarrollo permitió que otros profesionales además de los neurólogos pudieran interpretar el EEG. Esto también amplió las aplicaciones del EEG a estudio de isquemia cerebral, epilepsia y del nivel de actividad cerebral bajo anestesia general y sedación.

Existen para uso clínico en anestesia varios monitores de EEG digital como el BIS (índice biespectral monitor), Sed line, monitor de entropía, monitor de potenciales evocados auditivos y otros[12],[13],[14].

El Sed Line tiene algoritmos diferentes al BIS, que es el más validado a nivel internacional. Sin embargo, el Sed Line es equivalente al BIS y además muestra el espectrograma del EEG que cada vez cobra más importancia en la monitorización de la pro- fundidad anestésica. El espectrograma relaciona la frecuencia de las ondas cerebrales que presenta el paciente con el poder o cantidad de energía que presenta el espectro en cada orden de frecuencias representado con colores y los relaciona en una línea de tiempo.

EI PSI es el parámetro de EEG procesado que mide el monitor Sed Line con un algoritmo, lo que se correlaciona con el BIS durante la inducción, mantención y despertar de anestesia general. El valor de Sed Line adecuado durante la anestesia general es entre 25 y 50 . El espectrograma mide un valor llamado Spectral Edge frequency (SEF), que es el valor bajo el cual se encuentra el $95 \%$ de la potencia del espectrograma, es decir la mayor parte de la actividad cerebral en cada frecuencia. Durante anestesia general balanceada y anestesia endovenosa total, los valores adecuados de anestesia general medidos por SEF se encuentran entre 8 y 14[15],[16],[17].

La respuesta cerebral a cambios en las concentraciones de los anestésicos generales endovenosos y halogenados, es similar entre Sed Line y BIS[16].

Utilizando el BIS para monitorizar la profundidad anestésica, MTV Chang, observó una tendencia a la reducción de DP y DCPO al mantener BIS entre 40 y 60[9].

Moyce $Z$ en un metaanálisis sugiere que la anestesia BIS guiada disminuye el DP, pero no están claros los mecanismos para esto. Podría ser que el BIS permita administrar dosis anestésicas más adecuadas en pacientes más vulnerables[18].

No existen trabajos en población chilena o latinoamericana que hayan estudiado la incidencia de DP después de anestesia general en pacientes mayores monitorizados con BIS, Sed Line u otro monitor de EEG.

Debido a la mayor probabilidad que existe hoy en día de que los pacientes ancianos sean sometidos a cirugía debido al avance de la tecnología y a la necesidad de realizar nuevos procedimientos diagnósticos y terapéuticos bajo anestesia general, es importante enfatizar los factores que podrían prevenir complicaciones graves especialmente cerebrales como DP ya que sus consecuencias son siempre devastadoras. Si la monitorización con EEG ayuda a reducir la aparición de estas complicaciones cerebrales sería un gran avance en el manejo anestésico de los pacientes mayores[15],[2].

Este estudio utiliza el Sed Line y espectrograma para guiar la administración de un adecuado nivel de anestesia general, en pacientes > de 65 años, versus la administración de anestesia general guiada por los parámetros clínicos tradicionales hemodinámicos para estudiar la posibilidad de reducir la incidencia de DP.

\section{Objetivo general}

Determinar la incidencia de DP en pacientes mayores de 65 años luego de anestesia general con y sin monitorización cerebral de EEG.

\section{Objetivos específicos}

1.- Determinar la incidencia de DP en pacientes $>65$ años utilizando anestesia general guiada por monitor Sed Line vs anestesia general guiada por hemodinamia y signos clínicos. 


\section{Material y Métodos}

Se realizó un estudio clínico piloto con diseño de ensayo clínico randomizado, prospectivo, doble ciego.

\section{Criterios de inclusión}

- Pacientes mayores de 65 años.

- Pacientes sometidos a cirugía no cardíaca con anestesia general balanceada y endovenosa total.

- $\quad$ Pacientes con test preoperatorio MMT >24 (normal).

\section{Criterios de exclusión}

- Pacientes con daño cerebral de algún tipo como deterioro cerebral, demencia, Alzheimer, psicosis, alteraciones de lenguaje, auditiva o visual severas.

- Pacientes con Minimental test menor de 24.

- Pacientes sometidos a cirugía cerebral y cardíaca.

- Pacientes que se negaron a colaborar con el estudio[19].

Se seleccionaron 200 pacientes sometidos a anestesia general para cirugía no cardíaca ni cerebral, de más de 65 años, quienes ingresaron en forma consecutiva a Clínica INDISA de Santiago de Chile, desde noviembre de 2017 a abril de 2020 (Tabla 1). De los pacientes que ingresaron en forma consecutiva y que cumplían con los criterios de inclusión 19 declinaron participar en el estudio. Todos los pacientes seleccionados e incluidos en el estudio completaron los test y no se retiró ninguno del estudio por alguna causa (Tabla 2).

Tamaño muestral: Asumiendo una incidencia promedio de 20\% para DP, se determinó un tamaño de muestra mínimo óptimo de 195 pacientes por grupo para detectar una reducción del $50 \%$ en el riesgo de DP para una potencia de $80 \%$ y alfa $=$ 0,05 .

Debido a la llegada de la pandemia del coronavirus, se redujo dramáticamente el número de pacientes operados, por lo que decidimos detener el estudio y analizarlo como estudio piloto con los 200 pacientes que llevábamos en este punto.

Los pacientes se visitaron en su habitación para solicitarles su consentimiento escrito para participar en el estudio antes de ser llevados a pabellón y para realizar la evaluación preoperatoria y los test en forma tranquila.

Se randomizó a los pacientes a recibir anestesia general con monitorización EEG guiada (G1) o anestesia general con monitorización habitual sin EEG (G2). Se hizo asignación aleatoria secuencial en bloques descrita más adelante.

En el grupo 1 se instaló a los pacientes monitorización no invasiva habitual al llegar a pabellón, es decir, presión arterial no invasiva, oximetría de pulso y monitorización electrocardiográfica. Además, se instaló el sensor de SedLine (Massimo) en la frente de cada paciente. Se instaló monitorización invasiva según la cirugía y el estado de salud del paciente y el criterio del anestesiólogo tratante. Se ajustó el PSI entre 25 y 50, durante la mantención de la anestesia y el SEF (Spectral Edge Frequency) entre 8 y 14 . Si el valor de PSI era mayor a 50 o SEF mayor de 14, se aumentó la dosis de anestesia o analgesia según el criterio del anestesiólogo tratante y si el PSI era menor de 25 o SEF menor de 8 , se disminuyó las dosis de anestésicos administradas. Si había discordancia entre PSI y SEF, se privilegió el valor del SEF del espectrograma (Tabla 3). Si se produjo pattern de supresión de burst se disminuyó también la profundidad anestésica[9],[15].

\begin{tabular}{lccc}
\hline \multicolumn{4}{c}{ Tabla 1. Características demográficas } \\
\hline & $\begin{array}{c}\text { Grupo 1 } \\
\text { Con EEG }\end{array}$ & $\begin{array}{c}\text { Grupo 2 } \\
\text { Sin EEG }\end{array}$ & p \\
Número de pacientes & 99 & 101 & \\
Edad (años). Media $\pm \mathrm{sd}$ & $73,1 \pm 6,5$ & $72,9 \pm 6,4$ & 0,83 \\
Sexo femenino $\mathrm{n}(\%)$ & $42(42,4)$ & $50(49,5)$ & \\
Peso (kg). Media $\pm \mathrm{sd}$ & $72,1 \pm 12,8$ & $73,2 \pm 12,9$ & 0,56 \\
Talla (cm). Media $\pm \mathrm{sd}$ & $165,1 \pm 9,9$ & $164,2 \pm 9,5$ & 0,53 \\
IMC. Media $\pm \mathrm{sd}$ & $26,4 \pm 3,7$ & $27,0 \pm 3,9$ & 0,21 \\
\hline
\end{tabular}

\begin{tabular}{ccc} 
& Tabla 2 & \\
\hline & $\begin{array}{c}\text { Grupo 1 } \\
\text { Con EEG }\end{array}$ & $\begin{array}{c}\text { Grupo 2 } \\
\text { Sin EEG }\end{array}$ \\
ASA status. N $(\%)$ & $12(12,1)$ & $9(8,9)$ \\
1 & $71(71,7)$ & $80(79,2)$ \\
2 & $16(16,2)$ & $12(11,9)$ \\
\hline
\end{tabular}

\begin{tabular}{cccc}
\hline \multicolumn{4}{c}{ Tabla 3 } \\
Grupo 1 & Grupo 2 & p \\
\hline Duración anestesia (min) & $143 \pm 103,8$ & $144 \pm 100,5$ & 0,90 \\
\hline
\end{tabular}

La hemodinamia se corrigió con volumen, drogas vasoactivas de acuerdo con el criterio del anestesiólogo tratante.

En pacientes del grupo 2, al llegar a pabellón se les instaló el monitoreo no invasivo habitual de igual manera que en el otro grupo y el sensor de SL (Massimo) en la frente de cada paciente. La monitorización invasiva se hizo de acuerdo a la cirugía a realizar y al estado de cada paciente. La anestesia general se manejó con los parámetros clínicos habituales. La Presión arterial (PA) se mantuvo en rangos de $20 \mathrm{mmHg}$ sobre o bajo la basal, y la frecuencia entre 40 y 90 latidos por min. Cuando se observó signos de anestesia inadecuada como taquicardia, hipertensión, sudoración, deglución, movimientos, se aumentó la profundidad anestésica según el criterio del anestesista tratante. De igual manera se disminuyó la dosis anestésicas y analgésicas si se produjo hipotensión con bradicardia o sin ella, o se administró volumen y drogas vasoactivas como efedrina o fenilefrina.

Se midió PSI y espectrograma en ambos grupos, pero los anestesistas tratantes no tuvieron acceso al valor de éstos para el manejo de su anestesia en el grupo 2 (Tabla 4).

El sensor de SedLine se conectó al monitor Massimo en ambos grupos.

Los investigadores que realizaron los test eran ciegos al grupo que pertenecía cada paciente.

Las variables medidas fueron:

- Delirium PO: Se evaluó al paciente desde las 6 h posoperatorias y cada $12 \mathrm{~h}$.

- Se utilizó test Confussion Assesment Method (CAM)[20].

- Tiempo de duración de cada anestesia, datos demográficos de los pacientes y técnica de anestesia. 
- Se mantuvo la anestesia general en el grupo estudio entre los parámetros de Sed line entre 25 y 50 y sed Line entre 8 y 14 (Tabla 5).

\section{Aprobación Comité de Ética}

Se obtuvo la aprobación del estudio al Comité de Ética de la Facultad de Medicina de la Universidad Andrés Bello a la que pertenece la clínica en que se realizó el estudio.

Desde el punto de vista ético no se sometió a ningún paciente a riesgos debido a la investigación, ya que se manejó la anestesia general con drogas habituales en ambos grupos a criterio del anestesiólogo tratante y en el grupo manejado por EEG el control anestésico se hizo de la forma habitual, solo que se agrega un nuevo factor de control al análisis, que es el valor de PSI y SEF, lo que no expone al paciente a ningún riesgo adicional y existe la posibilidad de que sea un beneficio.

Todos los pacientes que participaron dieron su consentimiento firmado por escrito.

Se cuenta con todos los documentos de los pacientes, tanto los consentimientos como los test posoperatorios.

\section{Asignación de grupo de tratamiento}

Los pacientes seleccionados para este estudio se incorporaron a cada grupo mediante asignación aleatoria secuencial en bloques. Para este efecto, se utilizó una librería conocida como "blockrand", implementada en un software estadístico. Se asignó el grupo en sobres sellados[20],[21] (Tabla 6).

Tanto la estimación del tamaño de muestra como la asignación de los pacientes a cada grupo se realizó usando las librerías "pwr" [20] y "blockrand"[21] respectivamente. Estas rutinas están implementadas en el software estadístico R versión 3.3.1[22].

\section{Análisis estadístico}

Se definió como resultado primario la ocurrencia de DP con test Confussion Assesment Metod (CAM) positivo para DP los primeros 4 días posoperatorios.

El DP es de carácter discreto y dicotómico por lo que se evaluó utilizando el test exacto de Fisher. Variables continuas fueron comparadas entre grupos utilizando test de Student (ttest). Las evaluaciones estadísticas fueron realizadas utilizando el software $\mathrm{R}$ antes mencionado (Tabla 7).

\section{Resultados}

Se estudiaron 200 pacientes.

G1: Con EEG: 99 pacientes.

G2: Sin EEG: 101 pacientes.

Los pacientes en ambos grupos fueron comparables en edad, sexo, peso, estatura, ASA, duración de la anestesia. Todos estos parámetros no tuvieron diferencias significativas y se muestran en la Tabla 1.

El objetivo primario de incidencia de delirio fue de 7 pacientes en total. La incidencia de delirio en la población total fue de $3,5 \%$.

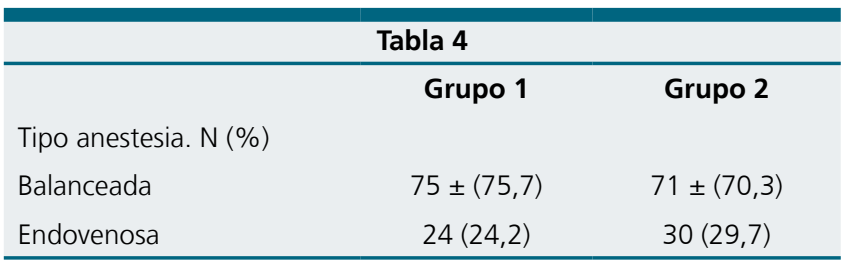

\begin{tabular}{lcc}
\hline \multicolumn{3}{c}{ Tabla 5. Cirugías en cada grupo } \\
\hline Procedimiento & Con & Sin \\
Cirugía digestiva & $36(36,4 \%)$ & $34(33,7 \%)$ \\
Cirugía general & $10(10,1 \%)$ & $15(14,9 \%)$ \\
Otorrino & $7(7,1 \%)$ & $5(5,0 \%)$ \\
Torácica & $4(4,0 \%)$ & $3(3,0 \%)$ \\
Traumatología & $24(24,2 \%)$ & $17(16,8 \%)$ \\
Urología no robótica & $11(11,1 \%)$ & $17(16,8 \%)$ \\
Urología robótica & $7(7,1 \%)$ & $10(9,9 \%)$ \\
\hline
\end{tabular}

\begin{tabular}{ccc}
\hline \multicolumn{4}{c}{ Tabla 6. Incidencia de DP según grupo } \\
\hline & CAM Si & CAM No \\
& Con DP & Sin DP \\
PSI & No & $\mathrm{Si}$ \\
Con & $98(99,0 \%)$ & $1(1,0 \%)$ \\
Sin & $95(94,1 \%)$ & $6(5,9 \%)$ \\
\hline
\end{tabular}

\begin{tabular}{ccc}
\multicolumn{3}{c}{ Tabla 7. Duración de anestesia y DP } \\
\hline CAM & Media & sd \\
No & 143,6 & 102,9 \\
Si & 151,4 & 72,44 \\
\hline
\end{tabular}

No existe diferencia significativa entre los promedios de duración de anestesia entre los pacientes delirio (CAM).

Los pacientes que presentaron delirio fueron 6 pacientes en el grupo 2 y 1 paciente en el grupo 1 .

Podemos entonces indicar que con base en los datos obtenidos existiría una reducción desde 5,9\% a 1,0\% cuando se utiliza PSI y espectrograma en el manejo anestésico. La diferencia entre ambas proporciones resulta ser no significativa ( $p$-value $=$ $0,1305)$

Un resultado concordante ( $p$-value) se obtiene con el test exacto de Fisher ( $p$-value $=0,1185$ ). El cálculo de potencia de este test indica 54\% de posibilidades de detectar una diferencia significativa con los tamaños de muestra utilizados.

Por tanto, la población estudiada no muestra diferencia significativa entre grupo 1 y 2 en la presentación de Delirio PO (Tabla 3), aunque es una tendencia importante la menor incidencia de delirio en el grupo 1, respecto del grupo 2.

Se reportaron 2 fallecimientos. Ambos en el grupo 2. Uno de 89 años, sexo femenino, probablemente por tromboembolismo pulmonar posoperatorio. El otro paciente de 72 años 


\begin{tabular}{llll}
\multicolumn{4}{c}{ Tabla 8. DP y ASA } \\
\hline CAM & 1 & 2 & 3 \\
No & $21(10,9 \%)$ & $146(75,6 \%)$ & $26(13,5 \%)$ \\
Si & Missing & $5(71,4 \%)$ & $2(28,6 \%)$ \\
\hline
\end{tabular}

No hay diferencia significativa entre ASA de pacientes con y sin DP.

con cáncer de próstata, insuficiencia renal, e hipertensión arterial, su causa de muerte se atribuyó a su cáncer de base (Tabla 8).

\section{Discusión}

El DP en pacientes mayores de 65 años después de cirugías es la más frecuente y la más devastadora complicación neurológica después de cirugía y anestesia[23],[24].

La mayor profundidad anestésica ha sido asociada a un aumento de DP[25],[26],[9].

EL DP tiene consecuencias deletéreas para los pacientes mayores y su entorno, que pueden ser muy graves. La prevención de estas entidades es muy importante no sólo para las familias y el propio paciente, sino que para el sistema de salud que se ve recargado en gastos por más complicaciones de estos pacientes con DP, más días de hospitalización de los pacientes y mayor posibilidad de que un paciente pierda su autovalencia.

Si bien en este estudio piloto no se alcanzó significación estadística entre grupos, se puede decir que tenemos una tendencia al menor DP en el grupo monitorizado con EEG.

En nuestros pacientes se administró anestesia general inhalatoria y endovenosa de manera similar en ambos grupos. Las cirugías realizadas también son de equivalente magnitud. Los grupos son similares en complejidad ya que no hay diferencias significativas en la clasificación ASA.

El análisis de los valores de PSI y espectrograma medidos en forma continua durante todo el proceso anestésico no se pudo realizar ya que no contamos con el sofware adecuado en el equipo que utilizamos en nuestro estudio, por lo que el análisis del efecto de nuestra monitorización fue mediante la aparición del delirium posoperatorio.

La incidencia de DP es menor que la reportada en la literatura mundial, ya que tenemos una incidencia de 3,5\% en el total de pacientes. Esto se puede deber a que las cirugías de nuestros grupos no son de gran complejidad, ya que en pacientes que van a unidades de cuidados intensivos la incidencia de DP puede ser entre $50 \%$ y $80 \%$, lo que no ocurrió en nuestros pacientes. También influye en la menor incidencia de DP el hecho de que los pacientes estén incluidos en un estudio, lo que hace que sean cuidados y monitorizados de manera regular por el personal del estudio además del personal de salud habitual de cada paciente.

La incidencia de DP en el grupo 1 es de $1 \%$ y en el grupo 2 es $6 \%$. Si bien hay diferencia en los números, no se alcanza diferencia significativa.

La razón de esta falta de significación puede ser el número de pacientes que no alcanzó un poder suficiente. Otra causa podría ser porque la mayoría de las cirugías no son de gran magnitud en las cuales la incidencia de DP es menor.

\section{Conclusión}

La monitorización con EEG de la anestesia general en cirugía no cardíaca no demuestra mejoría en la incidencia de DP en nuestro grupo de pacientes, aunque se logra una tendencia importante que apunta en este sentido. Deberemos esperar la oportunidad para completar este estudio aumentando el número de pacientes a estudiar.

Otro beneficio de este estudio es que logramos visibilizar el problema de la disfunción cerebral como probable complicación anestésica y hemos aumentado el interés de nuestros colegas anestesiólogos en el estudio y el uso de la monitorización cerebral, ya que este órgano es el blanco principal de nuestro quehacer anestésico.

\section{Referencias}

1. Billota el als. Trial $2011,12: 170$. Early postoperative cognitive dysfunction and postoperative delirium after anaesthesia with various hypnotics: study protocol for a randomised controlled trial: The PNOCCHIO trial. https://doi.org/10.1186/1745-6215-12-170

2. Mason SE, Noel-Storr A, Ritchie CW. The impact of general and regional anesthesia on the incidence of post-operative cognitive dysfunction and post-operative delirium: a systematic review with meta-analysis. Journal of Alzheimer's Disease 2010; 22(Suppl. 3): 67-79. https://doi.org/10.3233/JAD-2010-101086

3. Marcantonio ER. Postoperative delirium: a 76 years old woman with delirium following surgery. JAMA 2012; 308:73-81 https:// doi.org/10.1001/jama.2012.6857

4. Monk TG, Weldom BC, Garvan CW, et al. Predictors of cognitive dysfunction after major noncardiac surgery. Anesthesiology 2008; 108:18-30 https://doi.org/10.1097/01. anes.0000296071.19434.1e

5. Matthew T.V. Chan, MBBS, Benny CP Cheng et als. BIS-guided Anesthesia Decreases postoperative Delirium and Cognitive Decline. J Neurosurgical Anesthesiol, 2013; vol 25, N:1. https://doi. org/10.1097/ANA.0b013e3182712fba

6. Zhongcong Xie et als. The inhalation anesthetic isofluorane induces a vicious cycle of apoptosis and amyloid B- protein Accumulation. The Journal of Neurocience, 2007; 27(6): 1247-125 https:// doi.org/10.1523/JNEUROSCI.5320-06.2007

7. Jevtovic-Todorovic V, Hartman RE, Izumi $Y$, et al. Early exposure to common anesthetic agents causes widespread neurodegeneration in the developing rat brain and persistent learning deficits. Journal of Neuroscience 2003; 23: 876-82. https://doi. org/10.1523/JNEUROSCI.23-03-00876.2003

8. Ekman A, Lindholm ML, Lenmarken C and Sandin R. Reduction in the incidence of awareness using BIS monitoring. Acta Anaesthesiol Scand 2004; 48: 20-26 https://doi.org/10.1111/j.13996576.2004.00260.x

9. Chan MTV et als. BIS-guided Anesthesia Decreases Postoperative Delirium and Cognitive Decline. Neurosurg Anesthesiol 2013; 25:33-42 https://doi.org/10.1097/ANA.0b013e3182712fba

10. Newman S, Stygall J, Hirani S, Shashivadan S et als. Postoperative Cognitive Dysfunction after Noncardiac Surgery: A Systematic Review. American Society of Anesthesiologists, Inc 2007; 106(3): 423-430 https://doi.org/10.1097/00000542-200703000-00023

11. Rampil IJ. Electroencephalogram: In Albin MA, ed. Textbook of neuroanesthesia with neurosurgical and neuroscience perspecti- 
ves. New York: McGraw-Hill; 1997:193-219.

12. Rampil Ira J. A Primer for EEG Signal Processing in Anesthesia. 1998; Anesthesiology; 89:815-7. https://doi. org/10.1097/00000542-199810000-00002

13. Liu, Jin. Electroencephalogram Bispectral Analysis Predicts the depth of Midazolam induced sedation. Anesthesiology, 1996; 84:64-9 https://doi.org/10.1097/00000542-199601000-00007

14. White PF, Tang J, Ma H, Wender RH, Slonisky A and Kariger R. IS the patient state analyzer with the PSArray a Cost-effective alternative to the Bispectral Index Monitor during the perioperatice period?. Anesth Analg, 2004; 99:1429-35 https://doi. org/10.1213/01.ANE.0000132784.57622.CC

15. Poudon PL. Clinical Electroencephalography for Anesthesiologists Part I: Background and Basic Signatures. Anesthesiology, 2015;V 123: No 4

16. Emery R Brown, and Patrick $L$ Purdon. The aging brain and anesthesia. Curr Opin Anesthesiol. 2013; 26:414-419 https://doi. org/10.1097/ACO.0b013e328362d183

17. Escallier KE, Nadelson MR, Zhou D and Avidan MS. Monitoring the brain: processed electroencephalogram and peri-operative outcomes. Anaesthesia 2014; 69; 899-910. https://doi. org/10.1111/anae. 12711

18. Moyce Z, Rodseth $\mathrm{N}$ and Biccard BM. The efficacy of perioperative interventions to decrease postoperative delirium in non cardiac surgery; a Systematic review and meta-analysis. Anaesthesia 2014; 69: 259-269 https://doi.org/10.1111/anae.12539

19. Folstein MF, Folstein SE, McHugh PR.' 'Mini-mental state'. A practical method for grading the cognitive state of patients for the clinician. J Psychiatr Res 1975; 12: 189-198. https://doi. org/10.1016/0022-3956(75)90026-6

20. Stephane Champely Basic Functions for Power Analysis. (2016). R package version 1.2-0. https://CRAN.R-project.org/package=pwr

21. Greg Snow (2013) Blockrand: Randomization for block random clinical trials. R package version 1.3. https://CRAN.R-project.org/ package $=$ blockrand

22. R Core Team: A language and environment for statistical computing. R Foundation for Statistical Computing, Vienna, Austria. 2016 URL https://www.R-project.org/

23. J. Rodríguez Moretti. Cuidados quirúrgicos en Geriatría. Editorial Mediterráneo. Santiago, Chile. 2011.

24. Steinmetz J and Rasmussen LS. Peri-operative cognitive dysfunction and protection. Anaesthesia 2016; 71 (S1): 58-63 https://doi.org/10.1111/anae.13308

25. Wu CL, Hsu W, Richman JM, Raja SN. Postoperative cognitive function as an outcome of regional anesthesia and analgesia. Regional Anesthesia and Pain Medicine 2004; 29: 257-68. https:// doi.org/10.1097/00115550-200405000-00013

26. Radtke FM, Franck M, Lendner J, Kr€uger S, Wernecke KD, Spies CD. Monitoring depth of anaesthesia in a randomized trial decreases the rate of postoperative delirium but not postoperative cognitive dysfunction. British Journal of Anaesthesia 2013; 110(Suppl. 1): 198-105. https://doi.org/10.1093/bja/aet055

27. Inouye SK, van Dyck $\mathrm{CH}$, Alessi $\mathrm{CA}$, et al. Clarifying confusion: the confusion assessment method. A new method for detection of delirium. Annals of Internal Medicine 1990; 113: 941-8. https://doi.org/10.7326/0003-4819-113-12-941 